\title{
Developmental Toxicity of TCE Exposure in Zebrafish: Analyzing Vitamin D Metabolism
}

\author{
Briana M. Grisby ${ }^{1}$, Kathryn M. Thompson², Jennifer L. Freeman, $\mathrm{PhD}^{3}$ \\ ${ }^{1}$ Indiana University School of Medicine, ${ }^{2}$ Purdue University, School of Health \\ Sciences
}

Background and Hypothesis: Trichloroethylene (TCE) is an industrial solvent used since the 1940s. A known carcinogen, it is found in over half of Superfund sites where levels have reached 12,000 parts per billion ( $\mathrm{ppb} ; \mu \mathrm{g} / \mathrm{L}$ ), though the US Environmental Protection Agency maximum contaminant level in drinking water is $5 \mathrm{ppb}$. In this study, the zebrafish model was used to investigate the developmental toxicity of TCE by assessing its effects on metabolism. Preliminary studies have shown a reduction in gene expression of the cytochrome P450 (CYP) enzymes responsible for breakdown of vitamin D. As a result, we expected to see lower levels of downstream metabolites, namely calcitriol, as detected by an Enzyme-Linked Immunosorbent Assay (ELISA).

Experimental Design: At 0-2 hours post fertilization (hpf), embryos were dosed with TCE at either 0 or $10 \mathrm{ppb}$. At $72 \mathrm{hpf}$, the larvae were removed from the TCE and prepared for the ELISA procedure.

Results: ELISA revealed similar levels of calcitriol between the control and $10 \mathrm{ppb}$ groups. The difference between the two groups did not show statistical significance $(p>0.05)$.

Conclusion: These results suggest that there may not be a direct relationship between CYP gene expression and certain downstream metabolic effects or that the organism was able to compensate for the biological changes. If the latter, additional time points for TCE exposure could be evaluated. As studies continue, we plan to evaluate CYP gene expression at higher concentrations to correlate with other biological effects and determine if there is consistency with results from lower concentrations. 